\title{
Atrial Fibrillation and Stroke in Patients with Hypertrophic Cardiomyopathy: Important New Insights
}

\author{
Jeffrey S. Borer ${ }^{1}$ Dan Atar ${ }^{2}$ Thomas Marciniak ${ }^{3}$ \\ ${ }^{1}$ The Howard Gilman Institute for Heart Valve Disease and the Schiavone \\ Institute for Cardiovascular Research, State University of New York \\ Downstate Medical Center, Brooklyn, New York, United States \\ 2 Division of Medicine, Department of Cardiology B, Oslo University \\ Hospital and Institute of Clinical Sciences, University of Oslo, Oslo, Norway \\ 3 Bethany Beach, Delaware, United States \\ ${ }^{4}$ Department of Cardiology, Dong-A University, Busan, South Korea \\ ${ }^{5}$ Department of Neurology, Johns Hopkins University, Baltimore, \\ Maryland, United States
}

Thromb Haemost 2019;119:355-357.

Among the various non-ischaemic primary myocardial diseases recognized in humans, the most common is hypertrophic cardiomyopathy (HCM). Though first recognized more than 60 years ago because of the characteristic physical signs of its most dramatic form, idiopathic hypertrophic subaortic stenosis (IHSS), ${ }^{1}$ the rapid dissemination of the echocardiogram 10 years later soon led to the recognition that IHSS was merely a part of a continuum of genetically determined hypertrophic anomalies, often without outflow obstruction. ${ }^{2}$ The more characteristic haemodynamic abnormality is left ventricular diastolic dysfunction. This, in turn, can lead to left atrial dilatation which, if sufficiently severe, can contribute to the development of atrial fibrillation (AF).

Unless AF is recognized timely, the consequence frequently is left atrial thrombosis and embolization, leading to stroke or peripheral ischaemic sequelae. Thus, stroke prevention is central to the management of $\mathrm{AF}^{3}$ Indeed, in a tragic and dramatic irony, Andrew G. Morrow, who devised the most widely employed surgical approach to IHSS at the National Institutes of Health more than 50 years ago, ${ }^{4}$ developed $\mathrm{AF}$, was then found echocardiographically to have non-obstructive (i.e. inoperable) HCM, suffered a stroke and eventually died as a consequence.

The prevalence of AF has not been well defined in HCM, in part because of the difficulty in obtaining data from a sufficiently large and non-biased sample to ensure credibility. The study of Jung et al, published in the previous issue, ${ }^{5}$ provides some useful data concerning the extent to which HCM contributes to the global prevalence of AF.
Address for correspondence Victor Serebruany, MD, PhD, Department of Neurology, Johns Hopkins University, Baltimore, MD 21204, United States (e-mail: vserebr1@jhmi.edu).

Using a well-recognized national administrative database, these investigators provide a reasonable estimate of the prevalence of $\mathrm{HCM}$ among the Korean population with AF. Their more important finding was that the $\mathrm{CHA}_{2} \mathrm{DS}_{2}$-VASc score, commonly used to define risk of stroke in patients with AF and, therefore, a basis for the decision to prophylactically anticoagulate, has importantly different implications in patients with $\mathrm{AF}+\mathrm{HCM}$ versus patients with $\mathrm{AF}$ without HCM.

The reason for this discrepancy is not clear and requires further study. It is possible that thrombogenesis of the endothelium in HCM hearts is enhanced by outflow obstruction, though no assessment of this possibility is yet available. Another speculative possibility is that thrombosis-inducing anti-cardiolipin antibody (aCLa) is produced by some cell line in the HCM heart when AF occurs; such abnormal aCLa production is seen in patients with non-rheumatic aortic valve disease ${ }^{6}$ and, when present, appears to be associated with a proclivity for thromboembolization and stroke, though the reason for this association is unclear.

Other possible pathophysiological factors might be hypothesized. In any case, additional study of these potential pathways now is appropriate because of the findings of Jung et al. In addition, despite the now apparent risk of stroke, a distressingly low proportion of those with AF + HCM actually were being anticoagulated in Jung et al's cohort. This finding suggests the need to revisit the guidelines for management of AF for patients with HCM and to emphasize the need for early aggressive anticoagulation once $\mathrm{AF}$ is found. (c) 2019 Georg Thieme Verlag KG Stuttgart · New York
DOI https://doi.org/ $10.1055 / \mathrm{s}-0039-1678724$. ISSN 0340-6245. 
The incidence of AF in HCM has been inferred from observational data to be approximately $25 \%$ of patients first seen without $\mathrm{AF}^{7}$ However, a prospective assessment of the incidence of stroke in such patients has not been performed and is not available from the current study. The best available data were generated many years ago by a retrospective twocentre observational study involving 480 consecutive patients with HCM seen over 20 years and not managed according to any pre-set protocol or guideline. ${ }^{8}$ This study also revealed a $25 \%$ incidence of AF before the HCM diagnosis was made. After the diagnosis, $17 \%$ developed AF but the arrhythmia did not cluster in any temporal pattern. Rather, AF was found between 2 months and 29 years after the diagnosis and occurred at an average linearized rate of $2 \%$ per year. Further, in this series, stroke was unrelated to whether AF was paroxysmal or sustained. However, the relative risk of stroke in $\mathrm{HCM}+\mathrm{AF}$ was almost 18 -fold greater than among patients in the HCM cohort without AF. Coupled with the current data, these prior findings strongly suggest the appropriateness of long-term anticoagulation at the first evidence of AF.

A paucity of data also limits any conclusions about the utility of pharmacological anti-arrhythmic therapy, pulmonary vein isolation ('ablation') and the relative utility of these versus anticoagulation. Indeed, both the American College of Cardiology/American Heart Association/Heart Rhythm Society (ACC/AHA/HRS) guidelines for management of patients with $\mathrm{AF}^{9}$ and the parallel European Society of Cardiology (ESC) guidelines ${ }^{10}$ offer relatively little definitive guidance for patients with $\mathrm{HCM}+\mathrm{AF}$. Both recommend lifelong anticoagulation after development of $\mathrm{AF}$, though the ACC/AHA/HRS version specifically suggests anticoagulation irrespective of the $\mathrm{CHA}_{2} \mathrm{DS}_{2}$-VASc score. Jung et al's data support this suggestion because the risk of stroke in $\mathrm{HCM}+\mathrm{AF}$ in the absence of $\mathrm{CHA}_{2} \mathrm{DS}_{2}$-VASc risk factors was similar to the stroke risk in patients without HCM but with $\mathrm{CHA}_{2} \mathrm{DS}_{2}$-VASc score of 3 , generally considered a very strong indication for anticoagulation. Both American and European guidelines support attempts at rhythm control with pharmacological or ablation methods, though both appropriately note the absence of definitive studies to support this suggestion. Jung et al's data provide little guidance in this area and none with regard to the possible benefit of therapeutic reduction of outflow tract gradient when it exists (suggested without strong data by both guidelines for symptomatic patients with $\mathrm{HCM}+\mathrm{AF}$ ) because no pre-specified treatment protocol was employed in the study cohort and no randomization was attempted to facilitate data interpretation. Studies in this area now are needed.

In summary, the current study provides the most compelling data now available in support of a low threshold for lifelong anticoagulation in patients with $\mathrm{HCM}+\mathrm{AF}$ at the initial discovery of AF, even if only a paroxysm. This study also supports the need for prospective efforts to define factors that can predict AF development in HCM to allow earlier effective prophylactic therapy and to identify the factors which render the addition of HCM to AF more likely to cause stroke than AF in the absence of HCM. Finally, the potential use of direct oral anticoagulants (DOACs) instead of the vitamin $\mathrm{K}$ antagonists (e.g. warfarin) requires specific assessment. Though DOACs are far more convenient and, in theory, should be applicable in patients with HCM, the surprising finding of increased thrombogenesis and embolization with dabigatran in patients with prosthetic heart valves was unexpected, and has led to the contraindication of DOACs in the presence of valve prostheses. This issue will require direct study in $\mathrm{HCM}$, rather than assumptions based on pharmacological data. On the other hand, there is no reason to believe that DOACs will perform worse in HCM than warfarin, as recently postulated for yet another disease state: rheumatic valvular disease including mitral stenosis. ${ }^{11}$ After all, the HCM entity, in the previous ESC guideline on AF, was lumped together with rheumatic valvular heart disease, for the simple reason that a high embolic stroke rate was observed in both conditions. ${ }^{12}$ Indeed, in a separate publication, Jung et $\mathrm{al}^{13}$ have reported that when compared with warfarin, patients with $\mathrm{HCM}$ and AF on DOACs had similar stroke and major bleeding risks, but lower all-cause mortality and composite fatal cardiovascular events. Thus, patients with $\mathrm{HCM}$ and AF can be safely and effectively treated with DOACs.

\section{Conflict of Interest}

Dr. Atar reports personal fees from Boehringer-Ingelheim, BMS/Pfizer, Bayer Healthcare, MSD (Merck) and AstraZeneca, and grants from Medtronic and BMS, outside the submitted work. Dr. Borer reports personal fees from Takeda USA, during the conduct of the study; AstraZeneca, Novartis, ARMGO, Abbott Laboratories, Amgen, Servier, GSK, Pfizer and other from Biomarin, outside the submitted work.

\section{References}

1 Morrow AG, Braunwald E. Functional aortic stenosis; a malformation characterized by resistance to left ventricular outflow without anatomic obstruction. Circulation 1959;20(02):181-189

2 Henry WL, Clark CE, Epstein SE. Asymmetric septal hypertrophy. Echocardiographic identification of the pathognomonic anatomic abnormality of IHSS. Circulation 1973;47(02):225-233

3 Lip GYH, Freedman B, De Caterina R, Potpara TS. Stroke prevention in atrial fibrillation: past, present and future. Thromb Haemost 2017;117(07):1230-1239

4 Morrow AG, Brockenbrough EC. Surgical treatment of idiopathic hypertrophic subaortic stenosis: technic and hemodynamic results of subaortic ventriculomyotomy. Ann Surg 1961;154:181-189

5 Jung H, Yang PS, Sung JH, et al. Hypertrophic cardiomyopathy in patients with atrial fibrillation: prevalence and associated stroke risks in a nationwide cohort study. Thromb Haemost 2019;119 (02):285-293

6 Barbut D, Borer JS, Wallerson D, Ameisen O, Lockshin M. Anticardiolipin antibody and stroke: possible relation of valvular heart disease and embolic events. Cardiology 1991;79(02):99-109

7 Guttmann OP, Rahman MS, O'Mahony C, Anastasakis A, Elliott PM. Atrial fibrillation and thromboembolism in patients with hypertrophic cardiomyopathy: systematic review. Heart 2014;100(06): 465-472 
8 Olivotto I, Cecchi F, Casey SA, Dolara A, Traverse JH, Maron BJ. Impact of atrial fibrillation on the clinical course of hypertrophic cardiomyopathy. Circulation 2001;104(21):2517-2524

9 January CT, Wann LS, Alpert JS, et al; American College of Cardiology/American Heart Association Task Force on Practice Guidelines. 2014 AHA/ACC/HRS guideline for the management of patients with atrial fibrillation: a report of the American College of Cardiology/American Heart Association Task Force on Practice Guidelines and the Heart Rhythm Society. J Am Coll Cardiol 2014; 64(21):e1-e76

10 Kirchhof P, Benussi S, Kotecha D, et al; ESC Scientific Document Group. 2016 ESC Guidelines for the management of atrial fibrillation developed in collaboration with EACTS. Eur Heart J 2016;37 (38):2893-2962
11 Steffel J, Atar D. Non-vitamin K oral anticoagulants in 'valvular' atrial fibrillation: a call for action. Europace 2016;18(01): $1-3$

12 Camm AJ, Lip GY, De Caterina R, et al; ESC Committee for Practice Guidelines (CPG). 2012 focused update of the ESC Guidelines for the management of atrial fibrillation: an update of the 2010 ESC Guidelines for the management of atrial fibrillation. Developed with the special contribution of the European Heart Rhythm Association. Eur Heart J 2012;33(21): 2719-2747

13 Jung H, Yang PS, Jang E, et al. Effectiveness and safety of nonvitamin $\mathrm{K}$ antagonist oral anticoagulants in patients with atrial fibrillation with hypertrophic cardiomyopathy: a nationwide cohort study. Chest 2018. Doi: 10.1016/j.chest.2018.11.009 